Approach Used A project team, consisting of children's and adult palliative care providers, researchers and the All Wales Palliative Care Transition Lead, was convened to design a purposeful and effective training. The training was specifically created such that it would be suitable for a multi-disciplinary audience which would provide a comprehensive grounding if completed, but could also be delivered piecemeal so that participants had as much flexibility as possible regarding attendance. The content was informed by previous research.

Outcomes A series of six linked study days was designed such that each day could be attended as standalone, but those attending all of the days would have a comprehensive grounding in young adult care. Each study day includes a balance of clinical, practical and psychosocial topics suitable for a multi-disciplinary audience. Training will be delivered between June 2013 and June 2014.

Application to Hospice Practice The study day series is open to all professionals working in adult hospices who may increasingly be called upon to care for young people with life-limiting conditions. By improving the knowledge and skill base of these professionals, professional confidence will increase and lead to an improvement of care for these young people.

\section{P110 EVALUATION OF MEDICINES ADHERENCE IN DAY HOSPICE PATIENTS}

Julia Greenwood. St Gemma's Hospice, Leeds, England

10.1136/bmjspcare-2013-000591.132

Background Central to the care of patients in Day Hospice is an understanding of their compliance with medication. The Hospice has a Medicines Management Policy in place, but had no structured approach to measuring patients' levels of concordance, from their perspective.

Aim of the Evaluation The aim of the evaluation was to develop a method by which patient compliance could be assessed, explored and improved

Method The National Institute for Clinical Excellence (NICE) produced guidance and a patient questionnaire on Medicines Adherence in 2009. The questions explore the role of the healthcare professional in supporting patient's decision making, and understanding of their medicines. An amended version of the NICE questionnaire was developed and used, for which sixteen patients were randomly selected. Verbal consent was obtained and anonymity assured.

Results It provided useful information about our effectiveness in supporting patients with their medicines adherence. We scored well in engaging patients in joint decision-making, however some other aspects such as exploration of the burdens and benefits of medication, and common side effects needed improvement.

Limitations

Some questions could be misinterpreted, and there was no facility to expand on answers given. The length and style of the questionnaire was quite difficult to implement with people who were fatigued and unwell.

Conclusions This evaluation has been important to our understanding of our effectiveness in supporting medicines adherence. We intend to inform NICE of the amendments made prior to using the questionnaire within specialist palliative day services. We aim to further develop the questionnaire to evaluate patients' experiences and concerns regarding their medication regimes, and to address the need for carer involvement (End of Life Care Strategy 2008), as they underpin concordance for many of our patients.

\section{P111 TERMINAL OPIOID AND SEDATIVE TITRATION IN TWO HOSPICES}

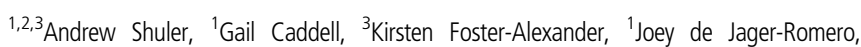
${ }^{2,3,4}$ Marlise Poolman. ${ }^{1}$ Nightingale House Hospice, Wrexham, Wales, ${ }^{2}$ Betsi Cadwaladr University Health Board, ${ }^{3}$ St Davids Hospice, LLandudno, WalesBangor University, Wales, ${ }^{4}$ Bangor University, Wales

\subsection{6/bmjspcare-2013-000591.133}

Introduction There was anecdotal observation by professionals working across both sites that there was a difference to practice with opioid and sedative titration at the end of life. As an extension to early work at one of the hospices, it was decided to consider practice across the region, so a second hospice was invited to participate.

Aims To quantitively assess practice of sedative use in relation to the EAPC recommended framework for the use of sedation in palliative care.

To quantitively assess practice of opioid use in the terminal phase.

To assess whether practice differs between hospices in North Wales, and to consider any potential reasons for the difference and implications for practice.

Methods A retrospective case-note review of patients who died in the two units. Data collected included drugs, doses and increments, and proxy assessment of symptoms in the last week of life (MSAS-GDI).

Results Groups were comparable in terms of background and demographics.

Practice differed with one unit using Midazolam and Levomepromazine more frequently and at higher starting doses.

There was no difference to symptom burden between sites.

Discussion All doses used were within the limits described in the EAPC framework.

At subsequent focus group discussion potential reasons were discussed including differences in anti-secretory medication use and its impact on sedative use.

These findings have precipitated further work on both sites.

\section{P112 MORPHINE AND OTHER OPIOID PAINKILLERS FOR MODERATE TO SEVERE PAIN: A NICE GUIDANCE COMPLIANT PATIENT INFORMATION LEAFLET}

${ }^{1,2}$ Ray Bunn, ${ }^{2}$ Amanda Gregory. ${ }^{1}$ Kamson's Pharmacy, Crawley, UK, ${ }^{2}$ St. Catherine's Hospice, Crawley, UK

\subsection{6/bmjspcare-2013-000591.134}

The Medicines Management Group (a multidisciplinary team comprising of medical, nursing and pharmacy representation) at an independent hospice has developed a patient information leaflet (PIL) on strong opioids. This decision was made in response to the recent National Institute for Health and Clinical Excellence (NICE) guidance on 'Opioids in palliative care: safe and effective prescribing of strong opioids for pain in palliative care of adults' ${ }^{(1)}$ which recommends that verbal communication between healthcare professionals and patients about their medicines should be supported by evidence based, written information. The aim was to produce a PIL which was NICE guidance 
compliant and which also contained other salient information relating to the use of strong opioids. The PIL is titled "Morphine and other opioid painkillers for moderate to severe pain" and contains the following section headings:

- Using Opioids - why prescribed, preparation and how to take, managing breakthrough pain

- Side effects - common side effects, what to do if experiencing side effects

- Driving and Travelling

- Other Information - collecting from pharmacy, opioids and alcohol, storage, disposal

There is also the facility on the front cover of the PIL for the dispensing pharmacy to stick duplicate labels of strong opioid medicines dispensed so that patients and carers know to which drugs the PIL refers. The PIL produced has, if locally adapted, wide potential for use in any palliative care organisation and pharmacy dispensing strong opioids for moderate to severe pain in the UK.

\section{P113 RE-DESIGNING HOW PATIENT MEDICINES INFORMATION IS PRESENTED ON DISCHARGE}

${ }^{1,2}$ Ray Bunn, ${ }^{2}$ Amanda Gregory. ${ }^{1}$ Kamson's Pharmacy, Crawley, UK, ${ }^{2}$ St. Catherine's Hospice, Crawley, UK

\subsection{6/bmjspcare-2013-000591.135}

A plethora of written patient information accompanies medicines dispensed (TTOs) for patients discharged from an independent Hospice inpatient unit. This includes:

- Manufacturer's patient information leaflets (PILs) for each medicine used within license - legally required.

- Locally developed PILs for medicines that are unlicensed or for use off-label.

- Green (regular) and blue (when necessary) 'Drug Information Cards' listing all medicines and dosage information electronically transposed from the TTO prescription.

- Locally developed PILs informing about medicine groups warranting special care in use e.g. 'morphine and other opioid painkillers for moderate to severe pain'.

It was observed that this 'jumble' of leaflets and medicines looked disorderly and unprofessional, with a real risk of information being lost, overlooked or simply discarded as unimportant. The aim was to develop how written patient information was presented to patients and carers in a way that was:

- Professional and distinctly noticeable.

- Neat, orderly and clear.

This problem was presented to the hospice Medicines Management Group (MMG) for resolution. The MMG is a multidisciplinary team comprising of medical, nursing and pharmacy representation with roles including the continuously improvement of the quality of pharmaceutical related practice and processes, often in an innovative way. They developed the following solution:

- 'Hospice branded' A4 front and rear cover, cardboard folder with rear cover hole punched near spine.

- Flat bar file fastener fitted to the rear page to hold:

- Explanatory page.

- A4 unlicensed PILs.

- A4 Drug Information Cards.
- An A4 plastic pouch holding the manufacturers PILs (variable sizes)

- The completed file is then placed in the bag containing TTOs.

This solution has potential for application in other settings.

\section{P114 IMPROVING THE USE OF PATIENTS OWN MEDICATION IN THE HOSPICE SETTING}

${ }^{1,2}$ Jeanette Crowther, ${ }^{1,2}$ Steven Wanklyn, ${ }^{1}$ Mel Johnson, ${ }^{1}$ Samantha Lund. ${ }^{1}$ Trinity Hospice, Clapham, London, ' Guy's \& St Thomas' NHS Foundation Trust London

\subsection{6/bmjspcare-2013-000591.136}

Introduction The use a of patients' own medications is improved at Trinity Hospice by patient's being encouraged to bring them in when being admitted. Advantages of using a patient's own medications are widely recognised and include aiding prompt completion of medicines reconciliation, thus achieving more accurate prescribing on admission, medication being available faster on the wards and of a quality that is suitable for self-administration, reducing the number of late and missed doses, patients continuing to use medication brands with which they are familiar or are required to due to inter-brand differences in bioavailability and considerable savings for the hospice's drug expenditure.

Aims

- To establish a procedure framework that improves the re-use of a patient's own medications whilst minimising patient risk.

- To inform governance priorities for the re-use of a patient's own medications particularly those involving high risk drugs.

Method A multidisciplinary team was established with representation from pharmacy, doctors and nurses in order to develop a clinical protocol for the management of a patient's own medications.

The protocol manages how a patient's own medications are assessed for suitability to be administered using a decision-support algorithm, obtaining further supplies and the essential documentation. Staff were deemed competent to undertake this procedure provided they attended a training workshop and successfully complete the training competency.

Results \& Discussion In order to quality-assure the protocol adherence to the process will be audited on a regular basis. The results of this alongside incident surveillance will provide ongoing risk management and identify areas for improvement.

Conclusion Trinity hospice has approved this protocol. Staff eligible to undertake a role have been accredited to do so. Under the terms of the Hospice's approval an audit of adherence and critical risk assessment will inform the further development of this medicine system.

\section{P115 CLINICAL PHARMACISTS - HANDS ON IN THE PATIENTS HOME AT END OF LIFE}

Joanne Bartlett, Louise Seager. John Taylor Hospice CIC, Birmingham, UK

\subsection{6/bmjspcare-2013-000591.137}

The introduction of frontline clinical pharmacy services for patients in their preferred place of care is redefining the 